\title{
Optimization of a compact static interferometer based on ImSPOC technology for a wide field polar lights monitoring
}

Etienne Le Coarer, Lucile Richard, Elisa Robert, Juana Rodrigo, Thierry Sequies, et al.

Etienne P. Le Coarer, Lucile Richard, Elisa Robert, Juana Rodrigo, Thierry Sequies, Frédéric Martin, Mélanie Prugniaux, Mathieu Barthelemy, Silvère Gousset, Jean Ravatin, Paul Monsinjon, Benoit Dupont, Bernard Godet, Emmanuel Roy, Jérôme Lacipière, "Optimization of a compact static interferometer based on ImSPOC technology for a wide field polar lights monitoring," Proc. SPIE 11852, International Conference on Space Optics ICSO 2020, 118521G (11 June 2021); doi: 10.1117/12.2599241

SPIE Event: International Conference on Space Optics - ICSO 2021, 2021, Online Only 


\section{International Conference on Space Optics-ICSO 2020}

Virtual Conference

30 March-2 April 2021

Edited by Bruno Cugny, Zoran Sodnik, and Nikos Karafolas
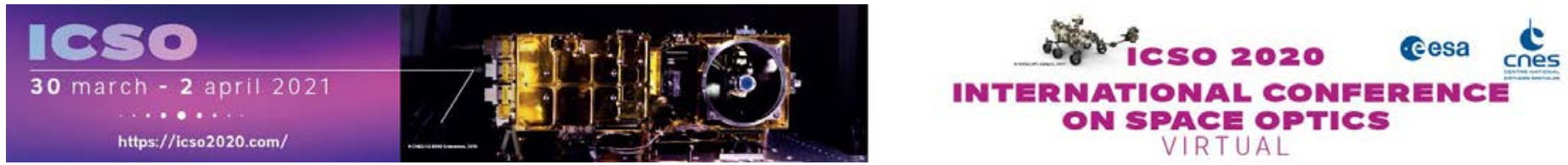

\section{Optimization of a compact static interferometer based on ImSPOC technology for a wide field polar lights monitoring}

\section{Cesa issopoceatings lecnes}




\title{
Optimization of a compact static interferometer based on ImSPOC technology for a wide field polar lights monitoring
}

\author{
Etienne le Coarer ${ }^{\mathrm{ab}}$, Lucile Richard ${ }^{\mathrm{a}}$, Elisa Robert ${ }^{\mathrm{b}}$, Juana Rodrigo $^{\mathrm{b}}$, Thierry Sequies ${ }^{\mathrm{ac}}$, \\ Frédéric Martin ${ }^{\mathrm{b}}$, Mélanie Prugniaux ${ }^{\mathrm{a}}$, Mathieu Barthelemy ${ }^{\mathrm{ab}}$, Silvère Gousset ${ }^{\mathrm{b}}$, \\ JeanRavatin $^{\mathrm{d}}$, Paul Monsinjon ${ }^{\mathrm{d}}$, Benoit Dupont ${ }^{\mathrm{d}}$, Bernard Godet ${ }^{\mathrm{d}}$, \\ Emmanuel Roy ${ }^{\mathrm{e}}$,Jérôme Lacipière ${ }^{\mathrm{e}}$ and Mathilde Heigeas ${ }^{\mathrm{e}}$.

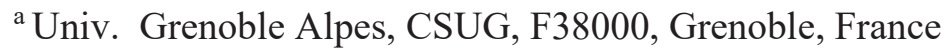 \\ ${ }^{\mathrm{b}}$ Univ. Grenoble Alpes, CNRS, IPAG, 38000 Grenoble, France \\ ${ }^{\mathrm{c}}$ Univ. Grenoble Alpes, IUT, 38000 Grenoble, France \\ ${ }^{d}$ Pyxalis, 170 Rue de Chatagnon, 38430 Moirans, France \\ ${ }^{\text {e } U n i v . ~ G r e n o b l e ~ A l p e s, ~ C N R S, N E E L-S E R A S, ~ 25, ~ a v e n u e ~ d e s ~ M a r t y r s, ~} 38000$ Grenoble, France
}

\begin{abstract}
In the context of the creation of a Distributed Space Weather Sensor System (D3S), the European Space Agency (ESA) is planning to fly a miniaturized payload for the monitoring of the auroral emissions with the project Wide Field Auroral Imager (WFAI). In order to meet ESA's requirements for an Auroral Optical Spectral Imager (AOSI), CSUG will build a breadboard as a proof of concept based on Imager Spectrometer on Chip (ImSPOC) technology.

Imaging Spectrometer on Chip (ImSPOC) technology consists of a matrix composed by multiple, low finesse, Fabry-Perot modules mounted in one unique structure. Each Fabry-Perot cavity has a fixed optical path difference distributed from optical contact up to maximum elevation in order to have the desired spectral resolution and the spectral coverage. So, in one snapshot it is possible to "scan" several optical path differences and reconstruct the spectrum for each point of the field of view. The advantage of this technology is to reduce the mass and dimensions but also that it collects sufficient number of photons allowing the observation of the faintest aurora (Not visible with naked eye) with short integration time. This makes it a particularly well-suited technology for small satellite payload design in the framework of polar lights monitoring.

We present here the optimization of the ImSPOC technology in the context of WFAI mission. This optimization allows us to recorded polar lights spectra in the wavelength range of 350 to $950 \mathrm{~nm}$ within an exposure between 10 and $60 \mathrm{~s}$. The field of view of the instruments is $57^{\circ} \times 57^{\circ}$, obtained by combination of 9 individual modules with small dimension (one module fits in a $60 \times 45 \times 55 \mathrm{~mm} 3$ box). The simulated performances of the instrument are really encouraging. The spectrometer must have a spectral resolution of $1 \mathrm{~nm}$ at $350 \mathrm{~nm}$.
\end{abstract}

Keywords: Imaging Spectrometer, Interferometer, Space weather, Fourier spectrometry.

\section{INTRODUCTION}

Wide Field Auroral Imager (WFAI) is a payload composed of two instruments (AUI and AOSI). The aim is to obtain full view of aurora oval from elliptical SSO orbits at every geomagnetic activity situations. Aurora Ultraviolet Imager (AUI) is a wide field imaging capability with three wide field cameras. Each of its camera has a dedicated narrow band filter in EUV (Extrem UltraViolet) domain $(130,135,140 \mathrm{~nm})$. Second instrument, Auroral Optical Spectral Imager (AOSI) described in this paper is composed by nine imaging spectrometer modules to achieve $1 \mathrm{~nm}$ spectral resolution in 350 $1080 \mathrm{~nm}$ visible and near infrared domain. Each of this module based on ImSPOC (Imaging Spectrometer On Chip) technology are identical pointing in different direction to fully cover $57^{\circ} \times 57^{\circ}$ field of view with a $5 \mathrm{mrad}$ sampling (i.e. 200x200 pixels). 
ImSPOC technology is a generic technique to obtain one full spectrum for each point in a field of view with an assembly of microlens array joined to a low finesse Fabry-Perot array [1].

While NanoCarb (same concept but focused on GHG detection) instrument in SCARBO H2020 program is the first instrument involving ImSPOC technology [2][3] in the infrared, AOSI in WFAI payload presented here is based on 9 ImSPOC modules. It allows to get a snapshot access up to $57^{\circ} \times 57^{\circ}$ field of view with $1 \mathrm{~nm}(@ 300 \mathrm{~nm})$ spectral resolution while similar instrument such hyperspectral camera is limited to $10 \mathrm{~nm}$ spectral resolution in the visible range.

\subsection{Motivation for this instrument}

Auroral emissions are one of the best tracer of the particle energetic inputs into the polar ionosphere [4] and references herein]. It allows reconstructing the electrons fluxes for energies up to $30 \mathrm{keV}$. Those particles classified as low energy particles are responsible for several detrimental effects of the particle precipitation into the polar atmosphere, mainly surface charging of satellite dielectric component or ground induce currents. They also are the main contributor to the auroral emissions.

However, one of the key goals of the auroral monitoring for space weather is to obtain the global energetic input into the entire ovals. It is then an important challenge to obtain a full or at least a large part view of the two auroral ovals. The oval size varies with geomagnetic activity, especially with $\mathrm{Kp}$ [5] but it is then reasonable to obtain a large view of the oval to design an instrument with a field of view of $57^{\circ} \times 57^{\circ}$ allowing to catch the entire oval when the activity is low.

The auroral emissions are line and band spectra. In the visible the main lines and bands are: the $\mathrm{O}^{1} \mathrm{~S}$ line at $557 \mathrm{~nm}$, the $\mathrm{O}^{1} \mathrm{D}$ triplet at respectively 630,636 and $639 \mathrm{~nm}$, the $\mathrm{N}_{2}{ }^{+}(0-1)$ band around $427 \mathrm{~nm}$ and the $\mathrm{N}_{2}$ first positive band in the $600-700 \mathrm{~nm}$ range. We can also notice the $\mathrm{N}_{2}{ }^{+}(0-0)$ band at $391 \mathrm{~nm}$ which presents a constant branching ratio with the $427 \mathrm{~nm}$ band.

If it could be possible to reach roughly the energetic input due to the electrons with a 3 colors RGB detector, it is clear that a full and precise estimation cannot be reached without extracting the full spectra at medium resolution of the auroral emissions. It is mainly due to the fact the main lines can show similar intensities for different inputs. A simple RGB detector necessitates some hypothesis like the distribution shape of the electron energy to allow a reconstruction of the electron flux and branching ratio between some vibrational branches of molecular bands.

This means that full spectra, on each pixel of the FoV is one of the only ways to allow a full reconstruction of the energetic input.

\section{INSTRUMENT DIMENSIONING}

\subsection{Hypothesis}

A first study allowing to dimension the instrument has been lead. The goal was to define 3 typical auroral conditions, faint, medium and strong and to calculate the intensity of each main line and band and check in which conditions these lines were visible by the instrument for different exposure times.

The simulations are done using the Transsolo code [6][7]. The Transsolo code is a kinetic simulation code which calculate from the atmospheric energetic inputs (Solar EUV flux and electrons precipitations) the transport of the particles along a vertical (or magnetic field line).

It allows from this transport calculation to deduce the collisions with atmospheric particles and thus the upper atmosphere emissions.

Three different conditions have been considered for the simulations: 


\begin{tabular}{|l|l|l|l|}
\hline & Faint & Medium & Strong \\
\hline Total energy $[\mathrm{keV}]$ & 0.3 & 2 & 7 \\
\hline Total energy [erg cm-2. $\mathrm{s}^{-1]}$ & 0.5 & 5 & 100 \\
\hline Column integrated green line $[\mathrm{kR}]$ & $\begin{array}{l}0.11 \\
\text { Non visible by naked eyes }\end{array}$ & 1.82 & 39.7 \\
\hline
\end{tabular}

Table 1. Auroral intensity for main AOSI lines.

\begin{tabular}{|l|l|l|l|l|}
\hline Line & $\begin{array}{l}\text { Wavelength } \\
(\mathbf{n m})\end{array}$ & $\begin{array}{l}\text { Intensity faint aurora } \\
(\mathbf{k R})\end{array}$ & $\begin{array}{l}\text { Intensity medium } \\
\text { aurora (kR) }\end{array}$ & $\begin{array}{l}\text { Intensity strong aurora } \\
\mathbf{( k R )}\end{array}$ \\
\hline O 1S & 557.8 & 0.110 & 1.82 & 39.7 \\
\hline N2+ 1N & 391 & 1.8 & 7.37 & 115.9 \\
\hline N2+ 1N & 427.8 & 0.63 & 2.58 & 40.56 \\
\hline O1D & $630,636,639$ & 0.1146 & 0.136 & 1.23 \\
\hline N2 1P & 670 & Full band: 0.21 & Full band: 2.10 & Full band: 43.02 \\
& & $\begin{array}{l}\text { Ratio for the 5-2: } \\
0.14^{*} 0.21=0.0294 \\
\text { Ratio: }\end{array}$ & $\begin{array}{l}\text { Ratio for the 5-2: } \\
0.14^{\star} 2.10=0.294 \\
\text { Ratio for the 5-2: }\end{array}$ & $\begin{array}{l}\text { Ratio for the 5-2: } \\
0.14^{*} 43.02=6.02\end{array}$ \\
\hline H alpha & 656 & $?$ & $\begin{array}{l}0.350 \\
\text { hypothesis from biblio } \\
\text { (ref needed) }\end{array}$ & $\begin{array}{l}\text { hypothesis from biblio (ref } \\
\text { needed) }\end{array}$ \\
\hline
\end{tabular}

Table 2. Auroral intensity for main AOSI lines.

\subsection{Partial synthetic spectra}

In order to infer the capabilities of the instrument, it is then fundamental to simulate a synthetic spectrum or at least a spectrum of the main lines or bands. The synthetic spectrum is constructed from Transsolo simulations in the same conditions. Lines are supposed to present gaussian shapes.

The width of the gaussian are chosen as follow:

The instrument will have a spectral resolution much worse than $10 \mathrm{~cm}^{-1}$. In order to be sure to simulate with a better simulation we choose to simulate the synthetic spectra with a resolution of $10 \mathrm{~cm}^{-1}$.

Line considered in these synthetic spectra are:

Green line $(557 \mathrm{~nm})$

- $\quad$ Red line $(630 \mathrm{~nm})$

- $\quad \mathrm{N}_{2}^{+}(427 \mathrm{~nm})$

- $\quad \mathrm{N}_{2}{ }^{+}(391 \mathrm{~nm})$

- $\quad \mathrm{N}_{2} 1^{\text {st }}$ positive band $(570-700 \mathrm{~nm})$

${ }^{1}$ Caution: 3 vibrational bands around at $670 \mathrm{~nm}$ as Reimei. Better band is the 5-2 (See below) 
$\mathrm{N}_{2} 1^{\text {st }}$ positive spectrum is difficult to simulate at this stage. REIMEI for example only considered 3 bands around $670 \mathrm{~nm}$ [9]. For this case, we consider only $\Delta \mathrm{v}=3$ and $\Delta=4$ branches because they are the most intense features of this band representing more than $80 \%$ of the full band. They are distributed between $570 \mathrm{~nm}$ and $700 \mathrm{~nm}(14286-17543 \mathrm{~cm}-1)$ as shown in the following Figure. These spectra are obtained in laboratory from the Planeterrella experiment (Bernard, PhD thesis, 2014).

Of course, these simulations do not represent full synthetic spectra but it gives an idea of the visibility of the main Auroral lines with the instrument.

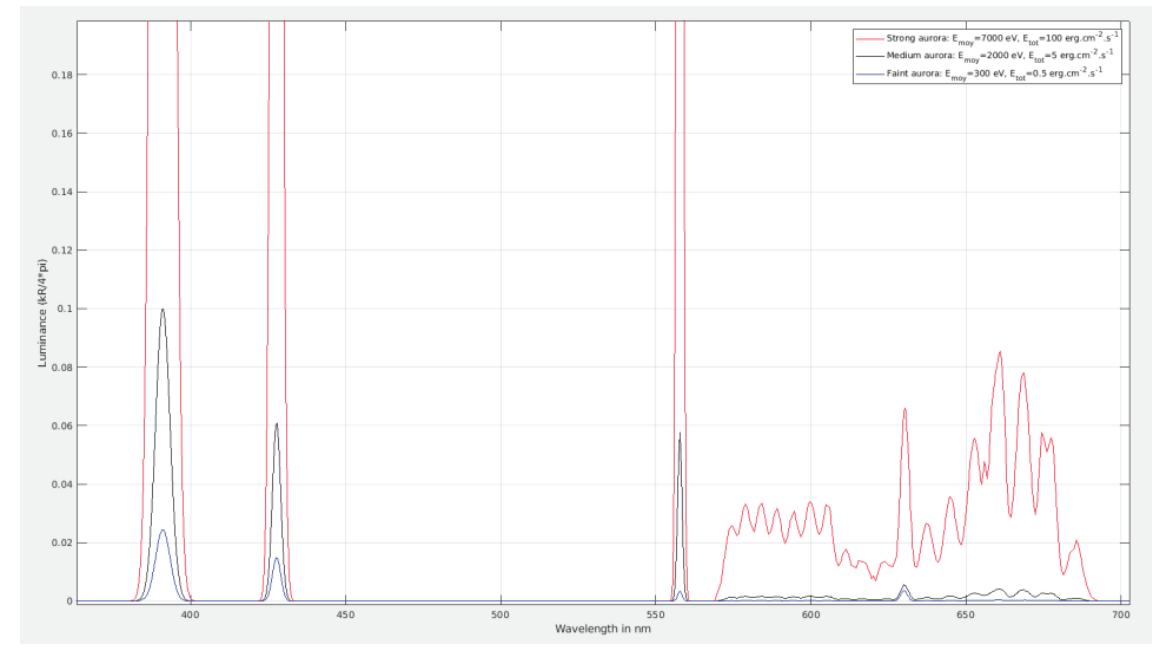

Figure 1. Synthetic spectra of the auroras for the three different conditions

\subsection{Moon comparison}

In the frame of the global monitoring of the aurora and its energetic inputs, one question is the visibility of the aurora in case of a moon period and with reflection on the ground on ice and snow or on clouds. We performed a study which establish then the intensity of the moon spectra as seen by the instrument.

As a first indication we performed calculation using rough hypothesis. The calculations have been done based on Cramer et al. [9] and considering the following hypothesis:

- Date of the data: 30 November 2012, 11:40:43. Almost full moon, 96\%

- Flux at the top of the atmosphere: $\sim 2.633 \mu \mathrm{W} \cdot \mathrm{m}^{-2} \cdot \mathrm{nm}^{-1}$ at $550 \mathrm{~nm}$ (Uncertainty $0.45 \%$ )

- Cloud albedo: 0.7 , No specular reflexion, reflexion in $2 \pi \operatorname{sr}$ (Lambertian)

- Nadir sighting.

These hypotheses are a worst-case observation since the phase is very high. It however could be worst in case of a possible specular reflection on the ground or on the clouds.

In order to calculate the effect of the moon on the visibility of the lines, we perform the following calculations:

The moon spectrum need to be integrated over the full moon spectrum and since it is a Fourier transform instrument, the total intensity will perturb the auroral measurements

The luminance of the moon $\mathrm{L}$ is given 


$$
L_{\text {moon }}=\int_{\lambda_{\text {Min }}}^{\lambda_{\text {Max }}} A \times F_{\text {Moon top }}(\lambda) \times \frac{\lambda}{h c} \times \frac{1}{2 \pi} d \lambda
$$

where $\mathrm{A}$ is the Albedo equal to $0.7, \mathrm{~F}$ the moon flux at the top of the atmosphere, $\lambda$ is the wavelength, $\mathrm{h}$ the Planck constant, and $\mathrm{c}$ the speed of light.

To simulate these effects, we produced full spectra of the moon light reflected on clouds or bright surfaces and partial synthetic spectra of the aurora.

The full moon spectra from 0.4 to $1 \mu \mathrm{m}$ is shown in figure 2 . The total intensity of the moon is $665 \mathrm{kR}^{2}$ or in luminance $52 \times 10^{9} \mathrm{ph} \cdot \mathrm{cm}^{-2} \cdot \mathrm{s}^{-1} \cdot \mathrm{sr}^{-1}$

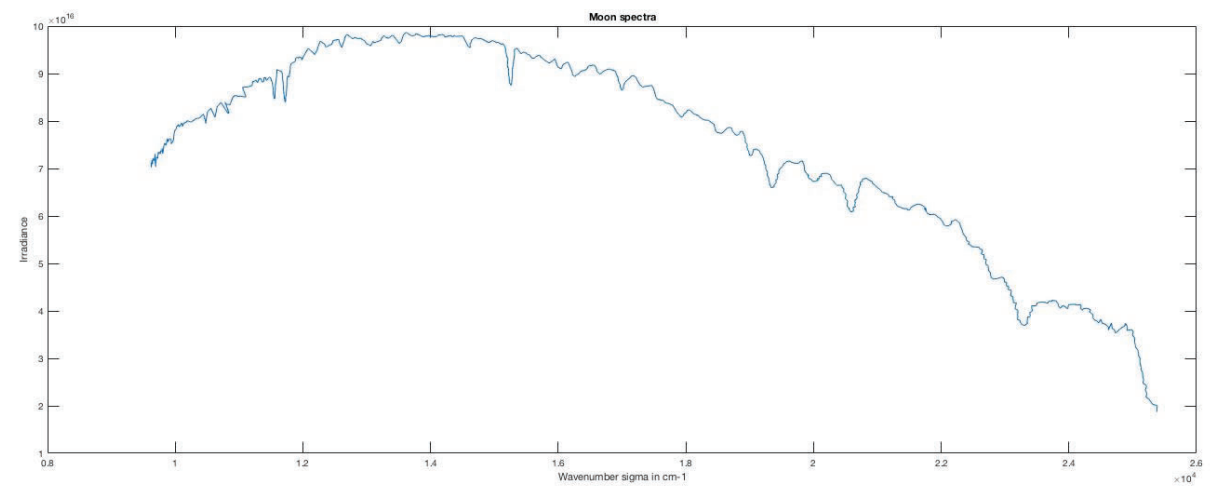

Figure 2. Moon full visible spectra from Cramer et al; 2013. (Note that irradiance expressed in $\mathrm{cm}-1$ not gives the Plank maximum with the same relationship given by classical Wien law).

\subsection{Geometrical factor and moon visibility}

\subsubsection{Geometry}

All these calculations must be mitigated by the geometry taking into account the cosine of two different angles. First, we have the angle between the region of interest and the moon orbital plane, and, in second, we have to consider the longitudinal angle between the satellite and the moon. This of course diminish the reflected intensity.

For example, at full moon, winter solstice with a local hour of $22 \mathrm{~h}$, one need to diminish the intensity of $\cos \left(30^{\circ}\right)$.

If the observed latitude is $70^{\circ}$, the angle is:

$\theta=70-23.4+5.14=51.7^{\circ}$,

where $23.4^{\circ}$ represent the inclination of the Earth Axis and $5.14^{\circ}$ is the angle between the lunar orbital plane and the ecliptic plane.

The geometrical factor is then $\cos \left(30^{\circ}\right) \times \cos \left(51.7^{\circ}\right)=0.53$ meaning the moon intensity is almost the half of the previously calculated intensity.

${ }^{2}$ A precise definition of the Rayleigh can be found in Baker (1974). One Rayleigh represents $10^{6} \mathrm{ph} . \mathrm{cm}^{-2} \cdot \mathrm{s}^{-1}$ distributed in $4 \pi \mathrm{sr}$ under the hypothesis of an isotropic flux. The luminance is obtained by dividing the intensity in Rayleigh by $4 \pi$ 
A full model of the reflected moon light is then need considering the full geometry of the observation. The simulation can be quite long to implement but is mandatory for further investigation. This simulation will be based on ROLO and POLO [11][12] codes which can reach $2 \%$ of precision for the full disk moon direct intensity. The albedo variation will be much more difficult to simulate since the cloud coverage is unknown and strongly variable.

\subsubsection{Visibility of the moon}

The orbit is planned to be a SSO orbit with perigee at $400 \mathrm{~km}$ and apogee at $4000 \mathrm{~km}$. Only the night side of the oval is visible, dayside is blinded by reflected solar light. As a first rough estimation, auroral oval will be visible only for solar zenith angle higher than $102^{\circ}$ perhaps a bit lower. This means that at the nadir less than the half of the auroral oval is visible. On the midnight-noon orbital plane; the moon is visible on the ground at the nadir, the half of the time from first quarter to last quarter through full moon. However, considering a field of view of $57^{\circ}$, a bit less than the half of the field of view is impacted by the moon but with much lower intensity. However, the geometrical factor will be very low.

The visibility will also depend of the albedo of the ground or of the clouds. There will be some differences regarding North and South poles. North pole shows more clouds but the ground albedo is often lower (Land or ocean coverage). There is less clouds in Antarctica but a large part of the oval will be above the ice cap with high albedo.

A rough estimation can be that the moon will be critical during 30\% of the time (close to full moon) and that $50 \%$ of the night side part of the ovals will be concerned by high albedo (It will depend of the width of the oval and thus of the Kp). This shows that the moon reflected light will be critical for around 10 to $20 \%$ of the observations which much less than the solar perturbation which represents more than $50 \%$ and is always critical in nadir observations.

\section{INSTRUMENT DESCRIPTION}

\subsection{ImSPOC principle}

ImSPOC technology is based on an observation of a field of view with a microlens array in front of 2D detector (figure 3 and 4). Each microlens is preceded by a small dedicated Fabry-Perot etalon which modulate images. To avoid image overlap between thumbnails, small walls forming small baffles are added between microlens array and detector. Baffles are black coated to avoid light reflection.
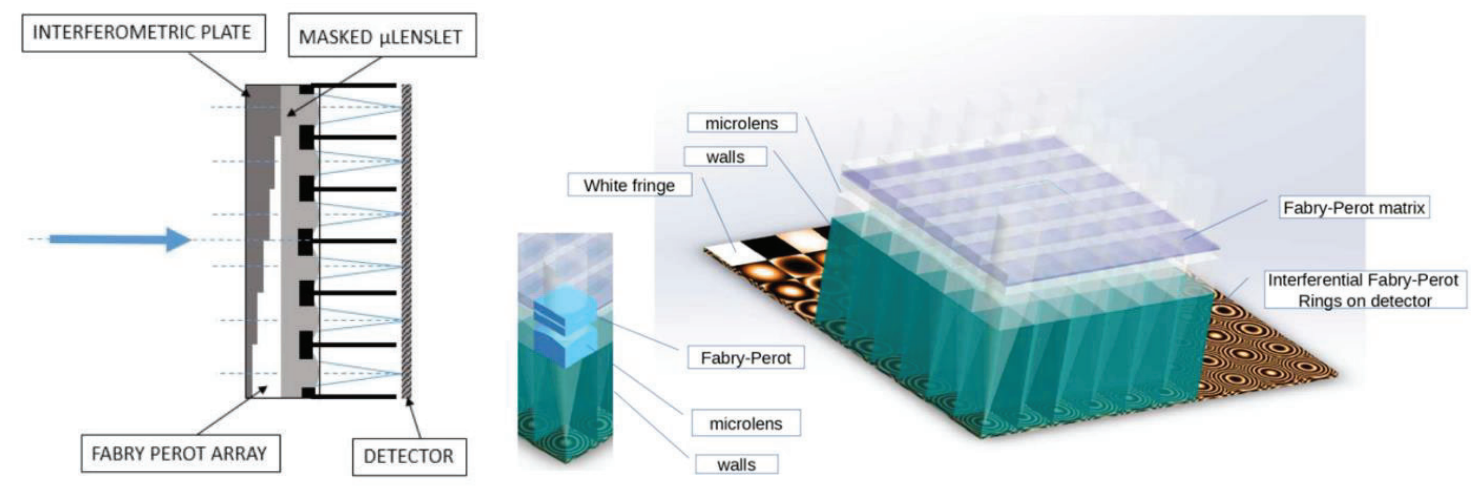

Figure 3. Left. AOSI instrument idea in a schematic: the light coming from the left pass through Fabry Perot Array compose by a two wave interferometer. The transmission of this interferometer is depending of the Fabry-Perot finesse estimated to $1 / \mathrm{F}(50 \%$ for $\mathrm{F}=2)$. Right. $3 \mathrm{D}$ view of ImSPOC.

Contrary to classical Fabry-Perot (FP) interferometry, here the finesse is low (between 2 and 10) in order to achieve a two waves modulation rather to filter light with narrow band filter as in classical FP etalons. 
Each FP of the matrix has a different spacing, for example distributed every $\lambda / 4$ if we want a fully sampled interferogram. For a given point in the field of view, the light intensity in different thumbnails provides an interferogram and its Fourier transform gives spectrum for this point. Thanks to that we are able to obtain a spectral image of the field of view observed.

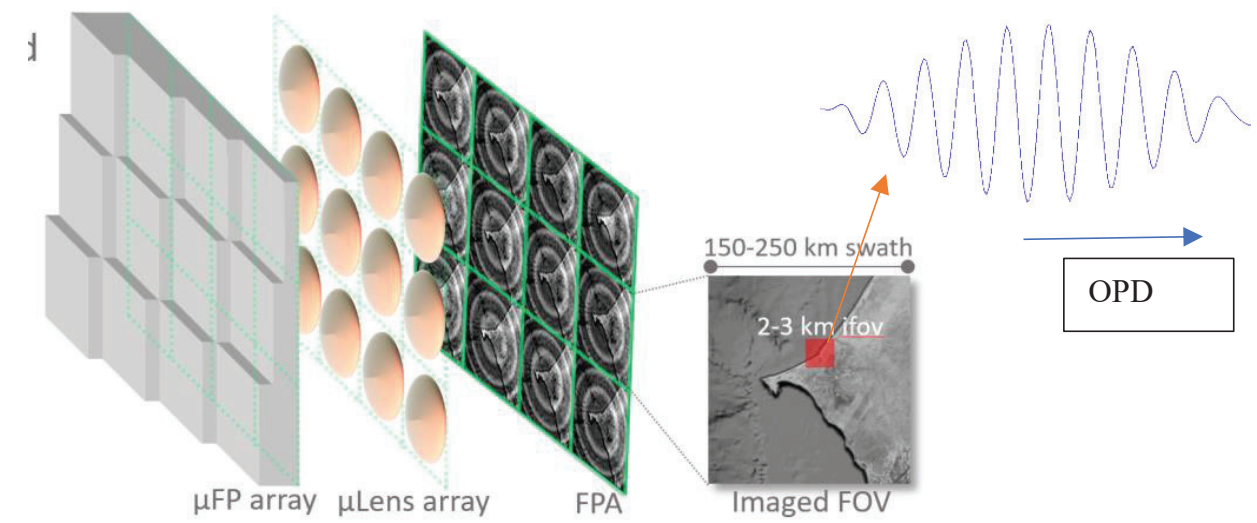

Figure 4. ImSPOC principle: for each microlens, the dedicated finesse $=2$ Fabry-Perot interferometer modulates light according its optical cavity spacing. The intensity sequence according OPD of equivalent point of the field of view in each thumbnail describes an interferogram.

\subsection{ImSPOC design}

The aim of ImSPOC is to give a high spectral resolution spectrometry in a wide field of view with the maximum of sensitivity. For that, focal lengths are very short with fast $\mathrm{f} / \mathrm{N}$. The limit corresponds to properly sample FP rings in image at highest FP spacing.
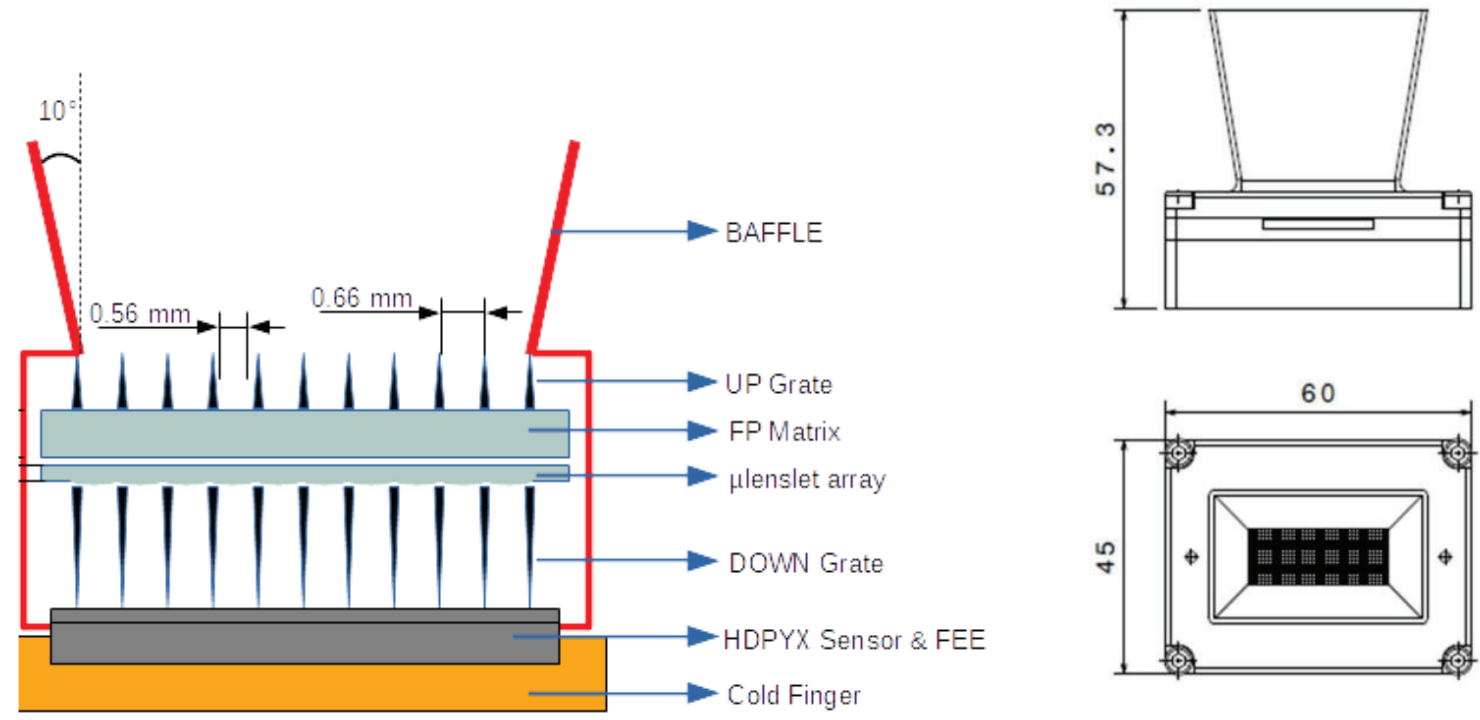

Figure 5. AOSI module architectural design 


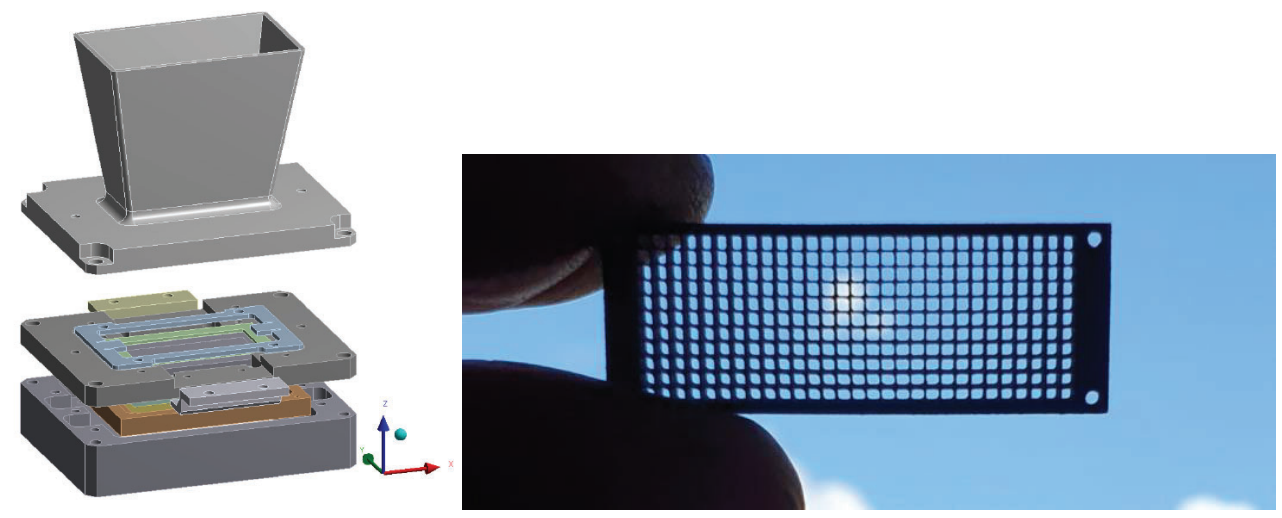

Figure 6. Detail of AOSI Module and example of black coated baffle grate issue from metallic 3D printing

\subsection{WFAI Payload description.}

As it was introduced, WFAI payload is composed by two instruments (AUI and AOSI). AUI is a wide field imaging instrument including 3 very small cameras observing at $130,135,140 \mathrm{~nm}$ in $60^{\circ} \times 60^{\circ}$ field of view covered by $1000 \times 1000$ pixels. Each camera is built with a 3 lenses objective lens in front of curved HDPyx Back-Side Illuminated CMOS sensor. Each of this camera take place in WFAI at the left side of the payload overview (see figure 7). In the same figure, 9 modules of AOSI occupy the right side of the image.

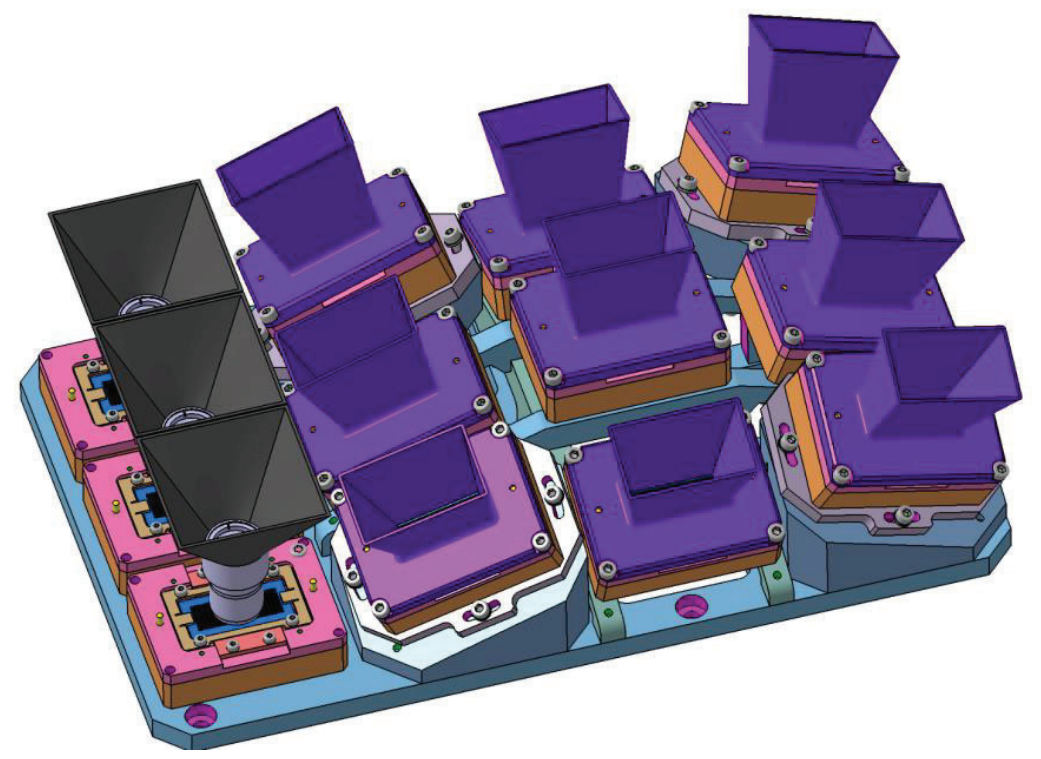

Figure 7. WFAI Payload overview. (left side) part, three EUV AUI $60^{\circ} \times 60^{\circ}$ field of view modules. (right side), 9 AOSI modules are set on 9 different directions in order to cover $57^{\circ} \times 57^{\circ}$ field of view. Size of payload: $280 \times 173 \times 100 \mathrm{~mm}$ including AUI payload. 


\section{INSTRUMENT PRECISE DIMENSIONING}

Because a $60^{\circ} \times 60^{\circ}$ field of view is not accessible with only one detector with such requirements, we decide to build an instrument with 9 modules of $20^{\circ} \times 20^{\circ}$ field of view with the following detector which has been selected for its very low readout noise with large pixel to insure larger sensitivity:

The sensor (HDPyx from Pyxalis) dimension parameters are:

- Detector size: 2808 x 1096 pixels

- Pixel size : $10 \mu \mathrm{m}$,

- $\quad$ Reading noise: $2.5 \mathrm{e}(\mathrm{RON})$

- Spatial resolution: $20 \mathrm{~km}$ at $2500 \mathrm{~km}$ altitude e.g. $10 \mathrm{~km} / \mathrm{pix}$, meaning 10/2500 $=0.004 \mathrm{rad}$ per pixel

With these characteristics, we could determine the focal length needed $\left(\mathrm{f}=10^{-5} / 0.004=2.5 \mathrm{~mm}\right)$.

The spectral resolution is estimated to $1 \mathrm{~nm}$ at $288 \mathrm{~nm}$ (note in Fourier, we define spectral resolution in $\mathrm{cm}^{-1}$ ). $\mathrm{R}$ is then 288 which is equivalent to a resolution of $120 \mathrm{~cm}^{-1}$.

Considering the ImSPOC concept, this means that the number of lens is 2 times the spectral power $\mathrm{R}=350$. The 700 lenses are then distributed over the whole detector splitting the image in 700 thumbnails. One thumbnail represents then $(2808 \times 1096 / 700)=4396$ pixels i.e. $66 \times 66$ pixels.

Considering the geometry of the detector, only $42 \times 16$ parts of $660 \mu \mathrm{m}^{2}$ are available. The lenses are distributed as $42 \times 16$ $\mu$-lenses i.e. 672 lenses. Despite this, we will in the following calculation consider that we will work with 700 lenses. The discrepancy is only of $4 \%$ and won't modify the main conclusions.

The square $\mu$-lenses area are then $435600 \mu \mathrm{m}^{2}$. So, for one $\mu$-lens, the optical etendue is then:

$\mathrm{S} \omega=0.66 \times 10^{-3} \times 0.66 \times 10^{-3} \times 0.004 \times 0.004=6.97 \times 10^{-12} \mathrm{~m}^{2} . \mathrm{sr}$

Since, one Rayleigh corresponds to $10^{10} \mathrm{ph} / \mathrm{m}^{2} / \mathrm{s}$ distributed in $4 \mathrm{x} \pi$ steradian, the equivalent luminance is then: $1 \mathrm{R} \Leftrightarrow$ $7.96 \times 10^{8} \mathrm{ph} \cdot \mathrm{m}^{-2} \cdot \mathrm{s}^{-1} \cdot \mathrm{sr}^{-1}$. For 1 second exposure time, $1 \mathrm{R}$ provides 0.00555 photons/s without taking into account the transmittance of the instrument. The total number of photon received by AOSI is 700 times this value equal to $3.88 \mathrm{ph} / \mathrm{s}$ or $233 \mathrm{ph}$ for $60 \mathrm{sec}$ exposure. With the transmission of instrument, we have 46.6 detected counts / R. The table 2 resume the number of photon at the entrance of the sensor for $60 \mathrm{sec}$ of exposure and for the different lines of faint, medium and strong aurora.

\begin{tabular}{|l|l|l|l|l|l|l|l|}
\hline & & \multicolumn{2}{|c|}{ Faint aurora } & \multicolumn{2}{c|}{ Medium aurora } & \multicolumn{2}{c|}{ Strong aurora } \\
\hline Line & $\begin{array}{l}\text { Wavelength } \\
(\mathbf{n m})\end{array}$ & $\begin{array}{l}\text { Intensity } \\
(\mathbf{k R})\end{array}$ & $\begin{array}{l}\text { 60s } \\
\text { exposure } \\
\text { (counts) }\end{array}$ & $\begin{array}{l}\text { Intensity } \\
(\mathbf{k R})\end{array}$ & $\begin{array}{l}\text { 60s } \\
\text { exposure } \\
\text { (counts) }\end{array}$ & $\begin{array}{l}\text { Intensity } \\
(\mathbf{k R})\end{array}$ & $\begin{array}{l}\text { 60s exposure } \\
\text { (counts) }\end{array}$ \\
\hline $\mathrm{O} 1 \mathrm{~S}$ & 557.8 & 0.110 & 5560 & 1.82 & 83720 & 39.7 & 1826200 \\
\hline $\begin{array}{l}\mathrm{N} 2+ \\
1 \mathrm{~N}\end{array}$ & 391 & 1.8 & 82800 & 7.37 & 339020 & 115.9 & 5331400 \\
\hline $\begin{array}{l}\mathrm{N} 2+ \\
1 \mathrm{~N}\end{array}$ & 427.8 & 0.63 & 28980 & 2.58 & 118680 & 40.56 & 1.865760 \\
\hline $\mathrm{O} 1 \mathrm{D}$ & $630,636,639$ & 0.1146 & 5272 & 0.136 & 6256 & 1.23 & 56580 \\
\hline
\end{tabular}




\begin{tabular}{|c|c|c|c|c|c|c|c|}
\hline & & \multicolumn{2}{|c|}{ Faint aurora } & \multicolumn{2}{|c|}{ Medium aurora } & \multicolumn{2}{|c|}{ Strong aurora } \\
\hline $\begin{array}{l}\text { N2 1P } \\
(5-2)^{3}\end{array}$ & 670 & $\begin{array}{l}\text { Full band: } \\
0.21 \\
5-2 \text { ratio: } \\
0.0294\end{array}$ & 9960 & $\begin{array}{l}\text { Full band: } \\
2.10 \\
5-2 \text { ratio: } \\
0.294\end{array}$ & 96600 & $\begin{array}{l}\text { Full band: } \\
43.02 \text { } \\
5-2 \text { ratio: } \\
6.02 \text { : }\end{array}$ & 1978920 \\
\hline $\begin{array}{l}\mathrm{H} \\
\text { alpha }\end{array}$ & 656 & $?$ & & $\begin{array}{l}0.350 \\
\text { hypothesis } \\
\text { from biblio }\end{array}$ & 16100 & $\begin{array}{l}1 \\
\text { hypothesis } \\
\text { from biblio }\end{array}$ & 46000 \\
\hline Total & & 2.864 & 131771 & 14.356 & 660376 & 241.41 & 11104860 \\
\hline
\end{tabular}

Table 2. Intensity of the aurora for different lines of interest and counts for a 60 s exposure

\section{PRELIMINARY RESULTS ON NGC7000, IC5070 \& IC 1318}

A very first $96 \times 96$ pixel ImSPOC module providing a $12^{\circ} \times 12^{\circ}$ field of view has been built on same $2808 \times 1096 \mathrm{HDPYX}$ 300 detector integrated in "Pyxalis camerakit". This first ImSPOC was put on a static photographic pod (Figure 8) pointing cygnus galactic HII complex region close to zenith co-adding 1s individual exposure during $1 \mathrm{~h}$. Because we used an uncooled detector running around $32^{\circ} \mathrm{C}$, we don't obtain very sensitive observations. Flat-field was acquired on twilight sky during sunset, dark acquisition was done during night with same detector temperature than observation and $650 \mathrm{~nm}$ laser pointer has been used for illuminating white screen during night in order to obtain wavelength calibration. Figure 8 shows an image on $650 \mathrm{~nm}$ laser illumination (right image). One can see on the right part of the image that low spacing Fabry-Perot images has no rings, at left, $28 \mu \mathrm{m}(350 \mathrm{~nm} / 4$ x 319$)$ spacing Fabry-Perot modulation gives rings.
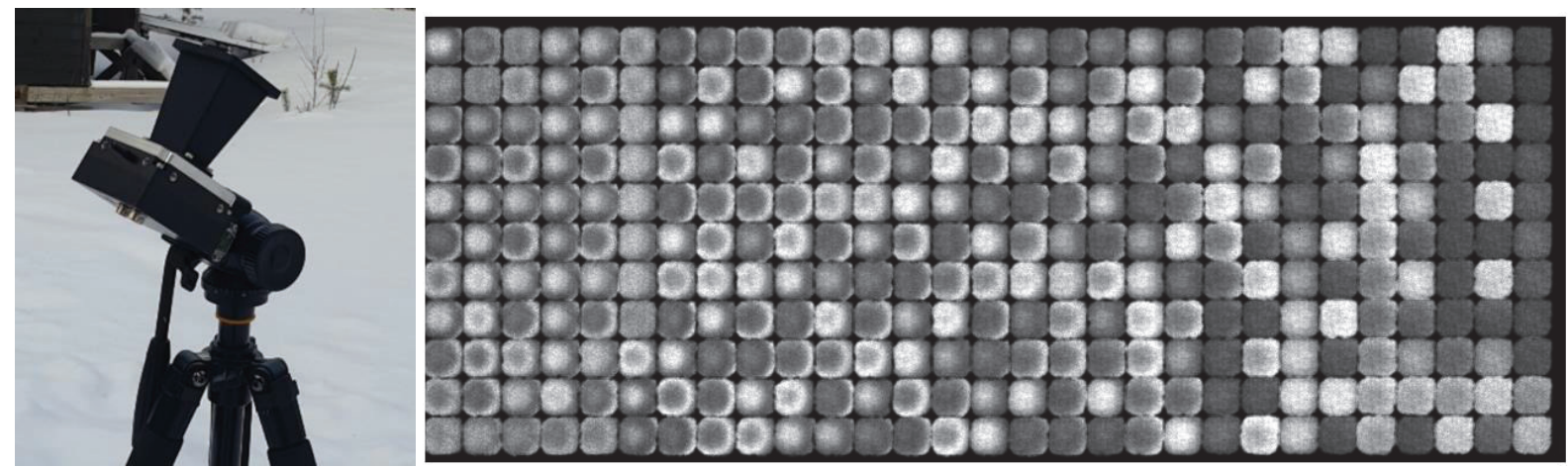

Figure 8. Left First ImSPOC prototype used to observe "America" galactic region NGC7000. Right: 650nm calibration image. In this image, from top to bottom and from right to left FP spacing are increasing by $350 \mathrm{~nm} / 4$ steps. 319 lenses are used.

Co-location of image was obtained using a Jupiter observation permitting us to center images with a 0.02pixel RMS error. Because Jupiter is very bright, one can see in figure 10 spikes on Jupiter image. This is coming from diffusion on baffles side. The level of diffusion is estimated less than $3 \%$ of the signal and a modelisation should below this contribution.

$35-2$ at $670 \mathrm{~nm}$ as Reimei 


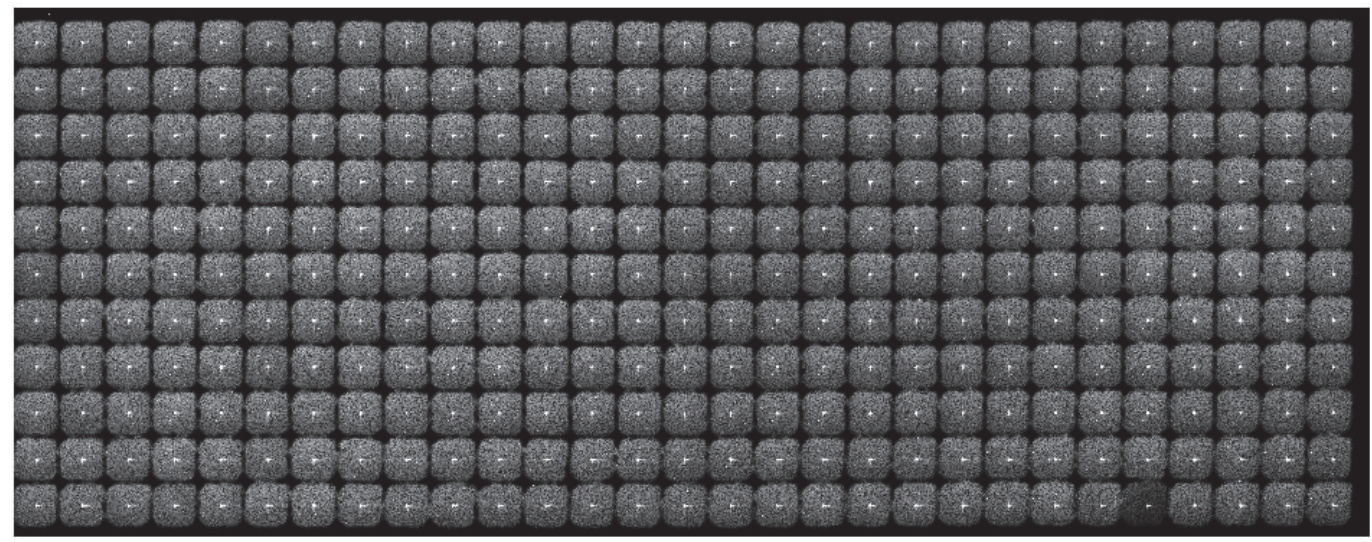

Figure 9. 1s exposure on Jupiter used to co-locate each thumbnails
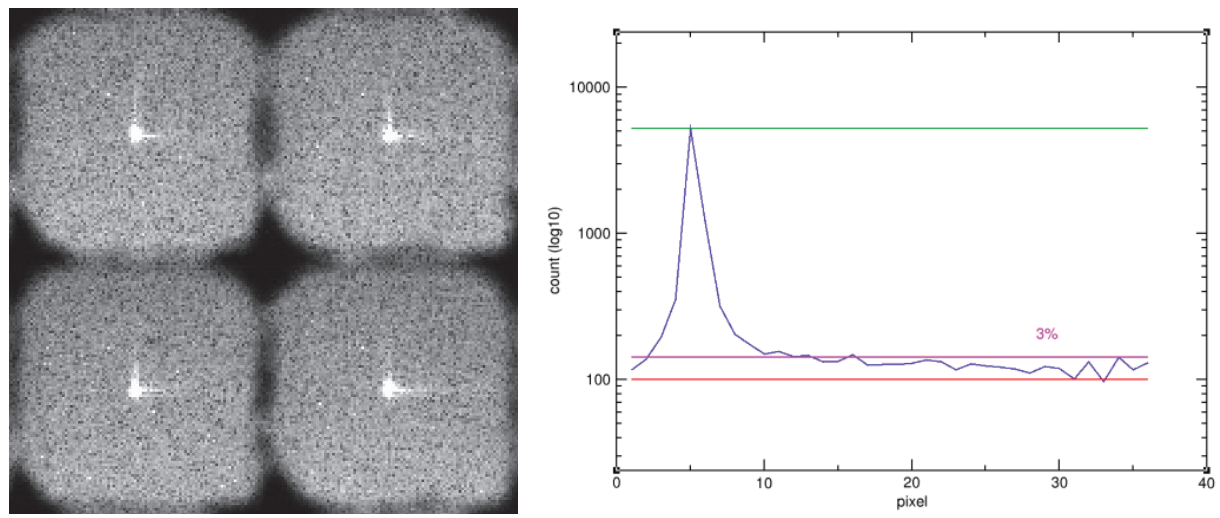

Figure 10.1s exposure on Jupiter. Zoom showing diffusion spikes due to reflection on side of baffle grate.
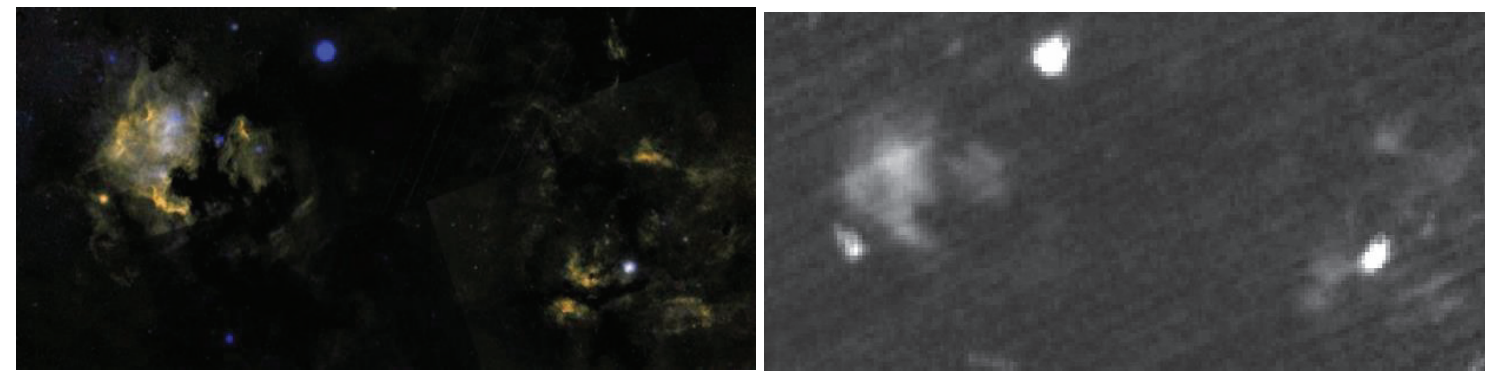

Figure 11. NGC7000 region. Left: Colorized DSS, Right: ImSPOC mosaiced field of view in $H \alpha$ line. 


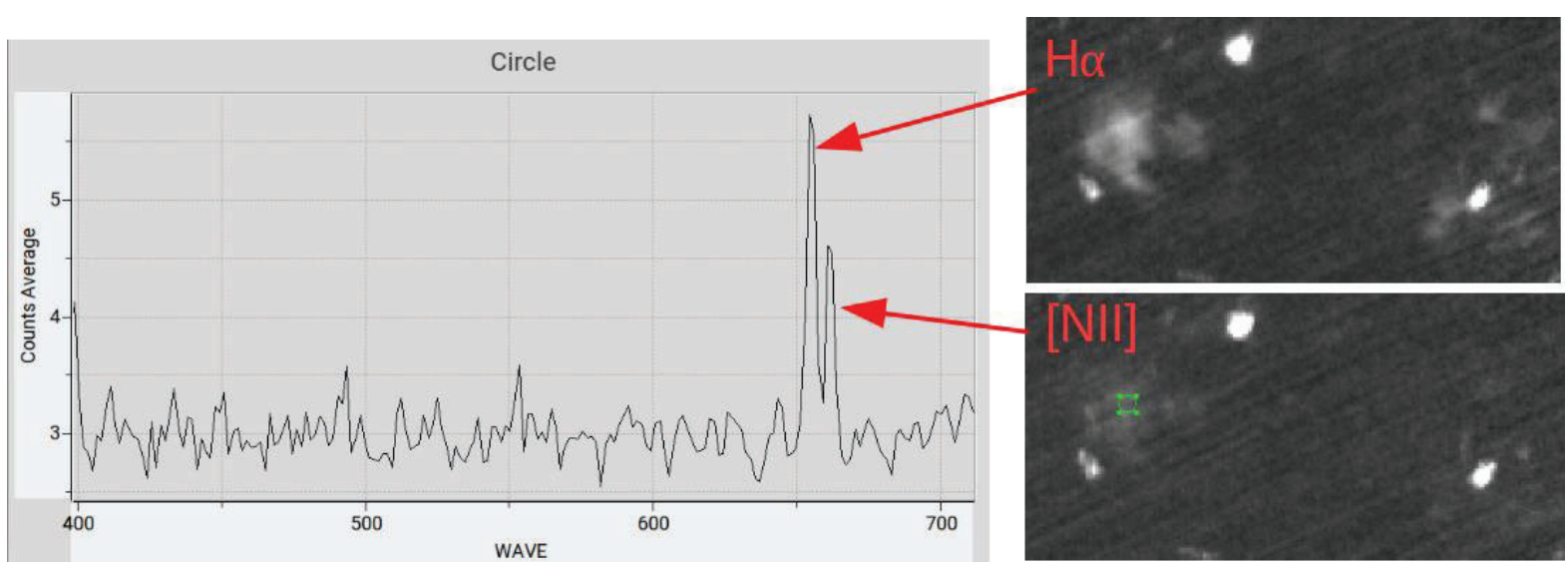

Figure 12. NGC7000 region in $656.3 \mathrm{H \alpha}$ line and 658.4 [N II] line.

\section{DISCUSSION}

The goal of this observation was to get raw estimation of the photometric sensibility in order to compare with aurorae brightness, which are more complicated to observe. An HII region are generally fainter than medium aurorae. We use the DEFPOS results [10] which gives HII region brightness in Rayleigh (see fig 13). Whole regions have brightness in the range between 300 and 1100R which corresponds to aurorae between faint (110R) and medium (1820R). A first drawback of such comparison come from the use of detector with a large difference of temperature where we just took an account about the evolution of dark with temperature. The difference in PRNU was not considered and the artifacts visible as lines following brooming. These artifacts have severe impact in detectivity and in future, the detector calibration will be done more accurately. Concerning the sensitivity threshold, the first ImSPOC was built with $\mathrm{f} / 5$ microlens and AOSI will have $\mathrm{f} / 3$ microlens winning a 3 factor on brightness.

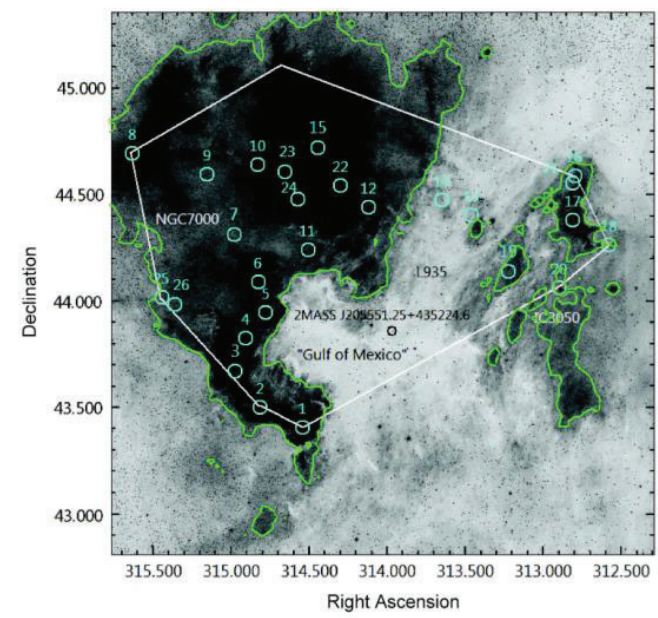

\begin{tabular}{cccccc}
\multicolumn{6}{c}{ Table 2 DEFPOS H $\alpha$ Observations from the W80 Nebula Complex } \\
\hline Region & $\begin{array}{c}\text { Intensity } \\
(\mathrm{R})\end{array}$ & $\begin{array}{c}\text { FWHM } \\
\left(\mathrm{km} \mathrm{s}^{-1}\right)\end{array}$ & $\begin{array}{c}V_{\mathrm{LSR}} \\
\left(\mathrm{km} \mathrm{s}^{-1}\right)\end{array}$ & $\begin{array}{c}V_{\text {HEL }} \\
\left(\mathrm{km} \mathrm{s}^{-1}\right)\end{array}$ & $\begin{array}{c}\log (\mathrm{EM}) \\
\left(\mathrm{pc} \mathrm{cm}^{-6}\right)\end{array}$ \\
\hline 1 & $1035.7 \pm 50.6$ & $52.9 \pm 1.7$ & $8 \pm 0.6$ & $-8.0 \pm 0.6$ & 3.4 \\
2 & $953.1 \pm 43.3$ & $53.1 \pm 1.5$ & $10.7 \pm 0.5$ & $-5.2 \pm 0.5$ & 3.3 \\
3 & $1031.7 \pm 37.7$ & $50.7 \pm 1.3$ & $12.5 \pm 0.4$ & $-3.4 \pm 0.4$ & 3.4 \\
4 & $692.6 \pm 29.6$ & $52.6 \pm 1.4$ & $11.3 \pm 0.5$ & $-4.6 \pm 0.5$ & 3.2 \\
5 & $846.3 \pm 31.9$ & $46.9 \pm 1.2$ & $12 \pm 0.4$ & $-3.9 \pm 0.4$ & 3.3 \\
6 & $974.7 \pm 38.1$ & $47.6 \pm 1.3$ & $10.9 \pm 0.4$ & $-5.0 \pm 0.4$ & 3.3 \\
7 & $436.8 \pm 27.4$ & $45.3 \pm 2.0$ & $10.6 \pm 0.7$ & $-5.3 \pm 0.7$ & 3.0 \\
8 & $594.5 \pm 30.6$ & $48.9 \pm 1.7$ & $4.9 \pm 0.6$ & $-10.9 \pm 0.6$ & 3.1 \\
9 & $424.8 \pm 34.7$ & $46.5 \pm 2.5$ & $6.7 \pm 0.9$ & $-9.2 \pm 0.9$ & 3.0 \\
10 & $721.1 \pm 39.6$ & $51.7 \pm 1.8$ & $4.4 \pm 0.6$ & $-11.5 \pm 0.6$ & 3.2 \\
11 & $586.7 \pm 35.4$ & $49.0 \pm 1.9$ & $9.3 \pm 0.7$ & $-6.7 \pm 0.7$ & 3.1 \\
12 & $813 \pm 34$ & $51.7 \pm 1.4$ & $8 \pm 0.5$ & $-8.0 \pm 0.5$ & 3.3 \\
13 & $342.9 \pm 32$ & $47.4 \pm 3.6$ & $5.9 \pm 1.1$ & $-10.2 \pm 1.1$ & 2.9 \\
14 & $259.8 \pm 26.5$ & $45.7 \pm 3.1$ & $5.5 \pm 1.1$ & $-10.6 \pm 1.1$ & 2.8 \\
15 & $1159.4 \pm 45.6$ & $54.7 \pm 1.4$ & $4.2 \pm 0.5$ & $-11.8 \pm 0.5$ & 3.4 \\
16 & $537.7 \pm 34.5$ & $50.7 \pm 2.1$ & $7 \pm 0.7$ & $-9.2 \pm 0.7$ & 3.1 \\
17 & $850.7 \pm 40.1$ & $55.5 \pm 1.6$ & $5.6 \pm 0.6$ & $-10.6 \pm 0.6$ & 3.3 \\
18 & $324.1 \pm 34.1$ & $52.2 \pm 3.7$ & $11.8 \pm 1.3$ & $-4.5 \pm 1.3$ & 2.9 \\
19 & $447.8 \pm 49.9$ & $48.8 \pm 4.0$ & $8.9 \pm 1.3$ & $-7.3 \pm 1.3$ & 3.0 \\
20 & $360.6 \pm 25.3$ & $48.2 \pm 2.2$ & $8.3 \pm 0.8$ & $-7.9 \pm 0.8$ & 2.9 \\
21 & $472 \pm 25.1$ & $48.9 \pm 1.7$ & $6.2 \pm 0.6$ & $-10.0 \pm 0.6$ & 3.0 \\
22 & $678.3 \pm 32.5$ & $49.9 \pm 1.5$ & $8.7 \pm 0.6$ & $-7.3 \pm 0.6$ & 3.2 \\
23 & $764.3 \pm 39.2$ & $48.6 \pm 1.6$ & $5.9 \pm 0.6$ & $-10.0 \pm 0.6$ & 3.2 \\
24 & $1019.9 \pm 47.4$ & $61.5 \pm 1.8$ & $11.2 \pm 0.6$ & $-4.8 \pm 0.6$ & 3.4 \\
25 & $290.2 \pm 29.8$ & $46.8 \pm 3.1$ & $5.2 \pm 1.1$ & $-10.6 \pm 1.1$ & 2.8 \\
26 & $351.2 \pm 29.3$ & $44.3 \pm 2.4$ & $8.5 \pm 0.9$ & $-7.3 \pm 0.9$ & 2.9 \\
\hline
\end{tabular}

Figure 13. from Aksaker[10], America and Pelican nebulae with identified region and corresponding brightness in table

\section{CONCLUSIONS}

AOSI and AUI take part of the WFAI Payload developed for D3S mission. This study permits us to develop a breadboard to verify performances of a very ambitious $350-1000 \mathrm{~nm} 200 \times 200$ imaging $1 \mathrm{~nm}$ spectrometer to cover 
$57^{\circ} \times 57^{\circ}$ field of view in a shoebox volume. Prior to test of the breadboard, we successfully use a ImSPOC pre-prototype on galactic HII region with brightness comparable with faint aurorae behavior.

\section{AKNOWLEDGMENTS}

This project is funding by ESA contract No. 4000127980/19/D/MRP (SSA P3-SWE-XX - Wide Field Auroral Imager). The first prototype of ImSPOC has been partially funded by AuRA region and FEDER under the project ImSPOC-UV. The FOCUS French label of excellence LabEx FOCUS (ANR-11-LABX-0013) contributes for ImSPOC concept development.

\section{REFERENCES}

[1] Guerineau, N., Le Coarer, E., Ferrec, Y., \& De La Barriere, F. (2020). U.S. Patent No. 10,677,650. Washington, DC: U.S. Patent and Trademark Office.

[2] Ferrec, Y., Bonnery, G., Brooker, L., Croizé, L., Gousset, S., \& Le Coarer, E. (2019, July). NanoCarb part 1: compact snapshot imaging interferometer for $\mathrm{CO} 2$ monitoring from space. In International Conference on Space Optics-ICSO 2018 (Vol. 11180, p. 1118021). International Society for Optics and Photonics.

[3] Gousset, S., Croize, L., Le Coarer, E., Ferrec, Y., Rodrigo-Rodrigo, J., \& Brooker, L. (2019). NanoCarb hyperspectral sensor: on performance optimization and analysis for greenhouse gas monitoring from a constellation of small satellites. CEAS Space Journal, 11(4), 507-524.

[4] Barthélemy, M., Kalegaev, V., Vialatte, A., Le Coarer, E., Kerstel, E., Basaev, A., ... \& Panasyuk, M. (2018). AMICal Sat and ATISE: two space missions for auroral monitoring.

[5] Sigernes, F., Dyrland, M., Brekke, P., Chernouss, S., Lorentzen, D. A., Oksavik, K., \& Deehr, C. S. (2011). Two methods to forecast auroral displays. Journal of Space Weather and Space Climate, 1(1), A03.

[6] Lilensten, J., \& Blelly, P. L. (2002). The TEC and F2 parameters as tracers of the ionosphere and thermosphere. Journal of atmospheric and solar-terrestrial physics, 64(7), 775-793.

[7] Vialatte, A., Barthelemy, M., \& Lilensten, J. (2017). Impact of Energetic Electron Precipitation on the Upper Atmosphere: Nitric Monoxide. The Open Atmospheric Science Journal, 11(1).

[8] Saito, H., Hirahara, M., Mizuno, T., Fukuda, S., Fukushima, Y., Asamura, K., ... \& Masumoto, Y. (2011). Small satellite REIMEI for auroral observations. Acta Astronautica, 69(7-8), 499-513.

[9] Cramer, C. E., Lykke, K. R., Woodward, J. T., \& Smith, A. W. (2013). Precise measurement of lunar spectral irradiance at visible wavelengths. Journal of research of the National Institute of Standards and Technology, 118, 396.

[10] Aksaker, N. (2013). DEFPOS H $\alpha$ observations of the W80 Complex. Research in Astronomy and Astrophysics, $13(5), 547$.

[11] Lacherade, S., Aznay, O., Fougnie, B., \& Lebégue, L. (2014, October). POLO: a unique dataset to derive the phase angle dependence of the Moon irradiance. In Sensors, Systems, and Next-Generation Satellites XVIII (Vol. 9241, p. 924112). International Society for Optics and Photonics.

[12]Xiong, X., Butler, J., Chiang, K., Efremova, B., Fulbright, J., Lei, N., ... \& Wu, A. (2014). VIIRS on-orbit calibration methodology and performance. Journal of Geophysical Research: Atmospheres, 119(9), 5065-5078. 